\title{
Analyzing the Fluid Flow of Transit-Time Ultrasonic Flowmeter with Image Processing Technique and Developing a Quality Metric Depending on Pipe Profile
}

\author{
M. A. GUNGOR
}

\begin{abstract}
The ultrasonic transit-time method measures the velocity and quantity of fluids in circular type pipes by using the difference of transit time between the ultrasonic pulses propagating with and against the flow direction. This method gives the average velocity of the fluid along a particular acoustic path. At least two ultrasonic transducers are used for an acoustic path. The multipath ultrasonic flowmeters have more acoustic paths. In this paper, the acoustic path between two transducers is described as pixels for the turbulent flow and formed a flow map for ideal flow conditions such as no elbow or bend depending on pipe profile. This map is used to analyze the fluid flow for different Reynolds numbers. Additionally, for any acoustic path between two transducers, the average fluid velocity is calculated using the obtained pixel values. Thus, a quality metric is developed in this paper. This metric calculates the ideal average fluid velocity ratio between the acoustic paths. The developed metric can be used to evaluate the quality of the ultrasonic flowmeter in the domain of turbulent flow.
\end{abstract}

Index Terms - Fluid Flow, Image Processing, Quality Index, Ultrasonic Flowmeter

\section{INTRODUCTION}

A CCORDING TO the measurement principle, the ultrasonic flowmeters are divided into two groups: Transit-Time Difference (TTD) and Doppler methods. The Doppler method is used for fluids containing particles such as bubbles and sand. This method relies on particles flowing through the fluid. Generally, this method is considered as having low performance due to the fact that the particle velocity is different from the fluid velocity. TTD ultrasonic flowmeter is used for homogeneous fluids that do not contain particles and has many advantages such as high accuracy, low maintenance and economical.

Many techniques have been developed to enhance the

MURAT ALPARSLAN GUNGOR, is with Department of Electrical and Electronics Engineering University of Hitit, Corum, Turkey,(e-mail: alparslangungor@hitit.edu.tr).

iD https://orcid.org/0000-0001-7446-7808

Manuscript received December 02, 2019; accepted June 03, 2020. DOI: $\underline{10.17694 / b a j e c e .654414}$ precision of TTD ultrasonic flowmeters, some of which find the optimal transducer angle [1], design the pipe [2], filter the signals $[3,4]$ and use FPGA [5, 6]. Zero-crossing and correlation methods are the most commonly used methods to find TTD [7]. Some of the scientists have studied to increase the "sensitivity for the zero-crossing method [8,9]. Leastsquare-sine-fitting technique is considered as an alternative method to obtain TTD [10].

To increase the accuracy in measuring, the multipath design has different acoustic paths. The calculation method for multipath ultrasonic flowmeter is described as follows: The fluid velocity is calculated for each path as in the one-path ultrasonic flowmeter. The average velocity is calculated by multiplying the calculated velocity value with the weight value for each path. The simplest way to calculate the weight value is the averaged method involving an equally weighted average of the path velocities [11]. In another method, the weight values are determined according to the geometric position of the transducers [12]. In this method, weight values are calculated by reference to the distance of the transducers from the pipe center. The transducer, which is close to the center, has a higher weight value. It has less weight value as it moves away from the center. Several path arrangement designs exist with weights to prescribe the position of acoustic paths, and an integration method is used such as Gauss-Jacobi and Optimal Weighted Integration for Circular Sections (OWICS) [13-15]. The major disadvantage of these methods is that weights are calculated based on fixed transducer positions. Researchers have focused on eliminating such disadvantages and increasing the accuracy by using a different algorithm such as Generalized Inverse Matrix, Levenberg-Marquardt, and variances of path velocities [16-18].

Although there are many studies on TTD ultrasonic flowmeters, research continues. This paper presents a flow map for ideal flow condition (FMIFC). FMIFC is formed in the domain of turbulent flow (i.e., Reynolds number (Re) > 4000) of transit-time ultrasonic flowmeter depending on pipe profile. Thus, researchers can analyze the fluid flow depending on Re. Furthermore, using FMIFC, a quality metric is developed. The developed metric calculates the ideal average fluid velocity ratio between the acoustic paths to obtain a reference value for ideal flow conditions. For the multiple 
acoustic paths, researchers can compare the application values with the reference values to evaluate their designs. In this paper, the following section presents the measurement principle of the transit-time ultrasonic flowmeter. FMIFC and the proposed quality metric are considered in Section III. Results and discussion are presented in Section IV. Finally, Section V concludes this paper.

\section{OPERATING PRINCIPLE OF TRANSIT-TIME ULTRASONIC FLOWMETER}

Due to the simplicity of the measurement principle, TTD method is often used in industrial applications [19]. At least two ultrasonic transducers are placed on the surface of the pipe. The transducers send ultrasonic pulses to each other. The fluid flow velocity is calculated by using the propagation time of the received pulses. The operating principle of the TTD method is shown in Fig. 1.

In Fig. $1, r_{0}$ is the pipe radius, $t_{A B}$ and $t_{B A}$ are the transit times, $\theta$ is the angle between the pipe and the direction of the ultrasonic wave and $\mathrm{v}_{\mathrm{m}}$ is the velocity on the center. In TTD method, the ultrasonic flowmeter measures the average velocity along the path between the ultrasonic transducers. Two ultrasonic transducers (shown as transducer $\mathrm{A}$ and transducer B in Fig. 1) send pulses propagating into and against the direction of the fluid flow. The transit time from transducer $\mathrm{B}$ to transducer $\mathrm{A}\left(\mathrm{t}_{\mathrm{BA}}\right)$ is greater than the transit time from transducer $A$ to transducer $B\left(t_{A B}\right)$ and they are calculated as follows:

$$
\begin{aligned}
& t_{A B}=\frac{L}{c+v \cos \theta} \\
& t_{B A}=\frac{L}{c-v \cos \theta}
\end{aligned}
$$

where $\mathrm{L}$ is the distance between the transducer $\mathrm{A}$ and transducer $\mathrm{B}, \mathrm{V}$ is the fluid flow velocity and $\mathrm{c}$ is the ultrasound speed in the fluid. The TTD is calculated as follows:

$$
\Delta t=t_{B A}-t_{A B}=\frac{L}{c-v \cos \theta}-\frac{L}{c+v \cos \theta}
$$

From the Eq. (3), we can obtain the v:

$$
\begin{aligned}
& v=\frac{L}{2 \cos \theta}\left(\frac{1}{t_{A B}}-\frac{1}{t_{B A}}\right)= \\
& \frac{r_{o}}{\sin \theta \cos \theta}\left(\frac{1}{t_{A B}}-\frac{1}{t_{B A}}\right)=\frac{r_{o} \Delta t}{\sin \theta \cos \theta t_{A B} t_{B A}}
\end{aligned}
$$

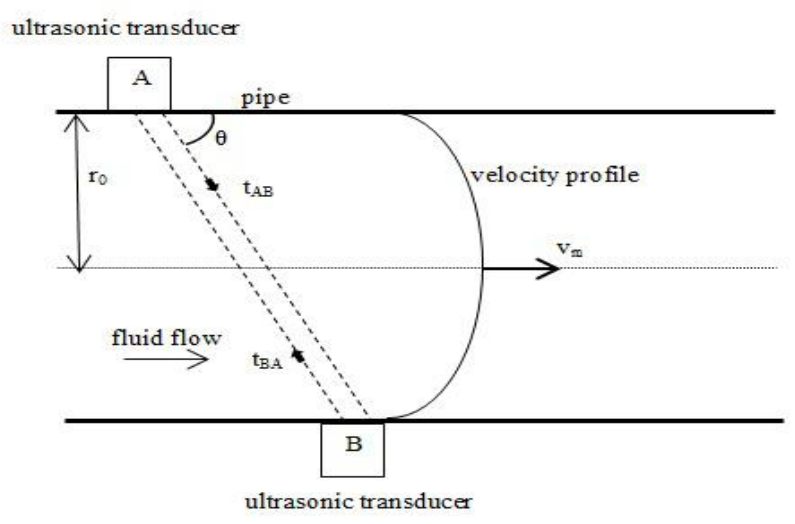

Fig.1. The principle of transit-time method

At low fluid flow velocities, the flow tends to be dominated by laminar flow, while at high fluid flow velocities flow is referred to as the turbulent flow. This paper is about the turbulent flow, i.e., $\operatorname{Re}>4000$. In the domain of turbulent flow, the velocity profile is called the "pipe profile" given by $[20,21]$ :

$$
v(r)=v_{m}\left(1-\frac{r}{r_{0}}\right)^{p}
$$

where $r$ is the distance from the center and $p$ is a parameter as chosen [21, 22]:

$$
p=0.25-0.023 \log _{10} \operatorname{Re}
$$

Remark that this parameter decreases with increasing Re and hereby $v(r)$ approaches to $v_{m}$. Therefore, the ratio of $v(r)$ to $v_{m}$ can be calculated depending on the Re.

\section{FMIFC AND THE PROPOSED QUALITY METRIC}

At first, in this paper, the fluid flow in circular type pipe for ultrasonic flowmeter application is described as pixels. The schematic of an ultrasonic flowmeter is shown in Fig. 2. The fluid flow direction in Fig. 1 corresponds to the $x$-direction (axial direction) in Fig. 2. The $y$ and $\mathrm{z}$ directions correspond to horizontal and vertical directions, respectively. When the fluid flow in Fig. 2 is described by pixels, the pixel values along the axial direction have the same values. Thus, the pixel values are obtained along the horizontal and vertical directions to describe the fluid flow in this paper.

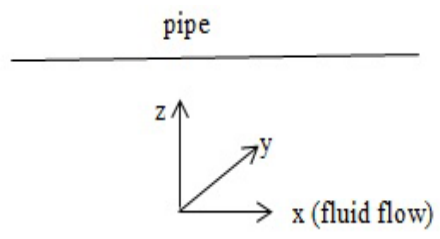




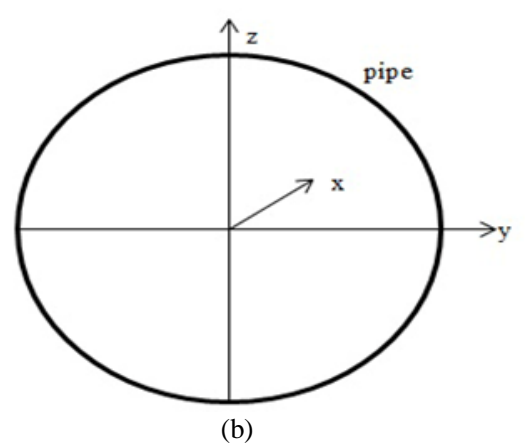

Fig.2. Schematic of an ultrasonic flowmeter (a) Longitudinal cross-section (b) Transverse cross-section

The pipe profile in Eq. (5) gives the velocity profile on the horizontal direction as shown in Fig. 1 and Fig. 2. If the ultrasonic transducers are placed on both ends of the y-axis shown in Fig. 2b, the acoustic path between these transducers can be described as pixels in Fig. 3 .

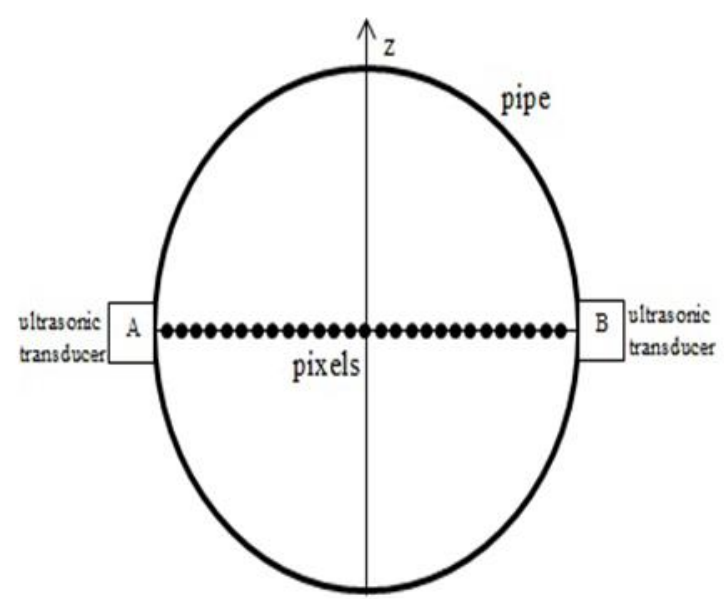

Fig.3. The acoustic path in the middle of the pipe

Any pixel value shown in Fig. 3 is between $0-255$ and depends on fluid velocity. The greater fluid velocity means the bigger pixel value. Each pixel value can be calculated using Eq. (5). $r=r_{0}$ corresponds to the pipe surface where the velocity equals zero $(\mathrm{v}(\mathrm{r})=0)$ and pixel value is zero. The pixels, which is close to the center, has a bigger value. $r=0$ corresponds to the center of the pipe where velocity is the maximum $\left(\mathrm{v}(\mathrm{r})=\mathrm{v}_{\mathrm{m}}\right)$ and pixel value is 255 . As mentioned above, for the larger $\mathrm{Re}, \mathrm{v}(\mathrm{r})$ approaches to $\mathrm{v}_{\mathrm{m}}$, resulting in bigger pixel value. After calculating each pixel value on the acoustic path, the average fluid velocity on the acoustic path can be obtained by averaging the calculated pixel values.

The velocity $\mathrm{v}$ given by Eq. (4), corresponding to the average fluid velocity on the acoustic path between the ultrasonic transducers shown in Fig. 3, can be obtained by using $\mathrm{v}_{\mathrm{m}}$ as follows [21]:

$$
v=\frac{v_{m}}{1+p}
$$

v values are calculated by using both pixels shown in Fig. 3 and Eq. (7) between $\mathrm{Re}=4000$ and $\mathrm{Re}=1000000$. The obtained values are normalized with $\mathrm{v}_{\mathrm{m}}$ and shown in Fig. 4. Instead of handling the entire distance between the two transducers, shown in Fig. 3, only the pixels between the ultrasonic transducer A and center are handled to avoid extra processing.

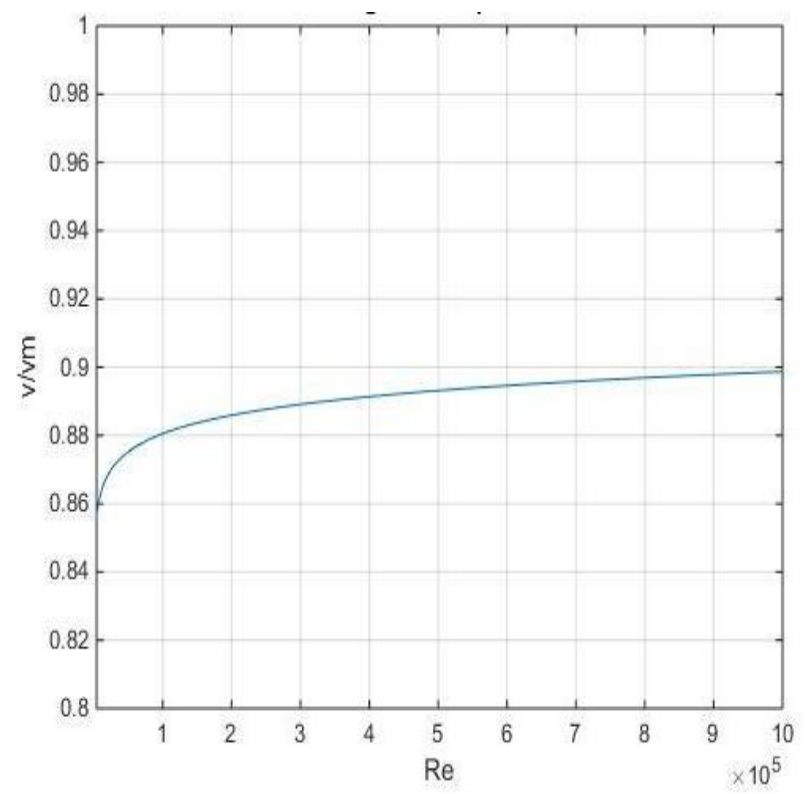

(a)

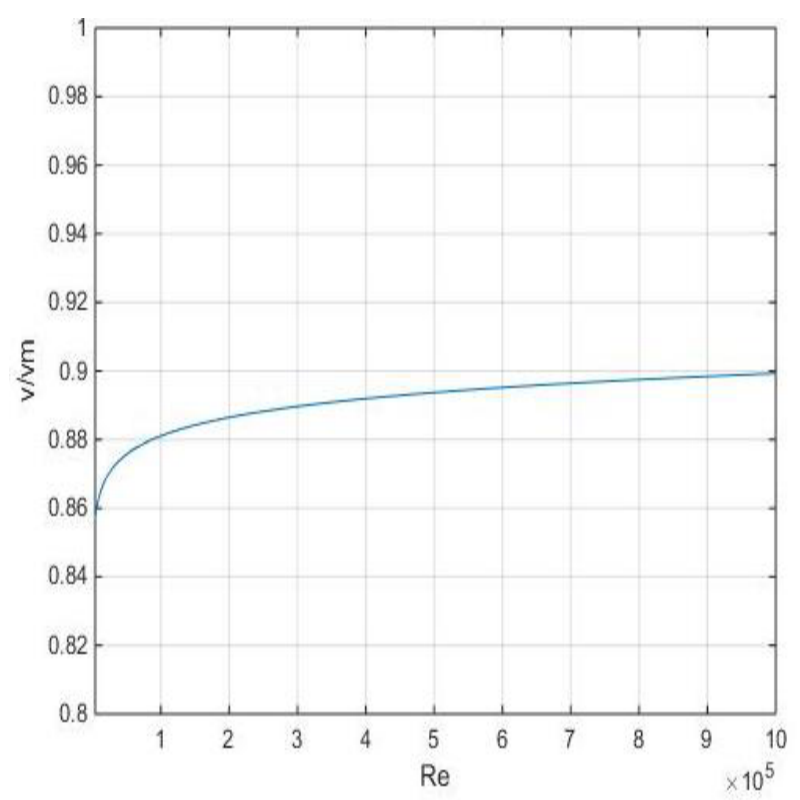

(b)

Fig.4. Obtained normalized average flow velocity vs. different Re values on the acoustic path in the middle of the pipe by using (a) Pixels (b) Eq. (7)

As shown in Fig. 4b, the normalized $v$ value for the $\mathrm{Re}=$ 4000 is 0.856 . It is approaching $\mathrm{v}_{\mathrm{m}}$ as the Re increases, and this value is 0.899 for the $\mathrm{Re}=1000000$. Comparing Fig. $4 \mathrm{a}$ and Fig. $4 b$, the normalized values obtained by pixels and Eq. (7) are equal to each other. If we consider the acoustic path in Fig. 3 as in Fig. 5, the pixel values of the acoustic path between each ultrasonic transducer pair and average flow velocity for 
the corresponding acoustic path can be calculated by the method mentioned above.

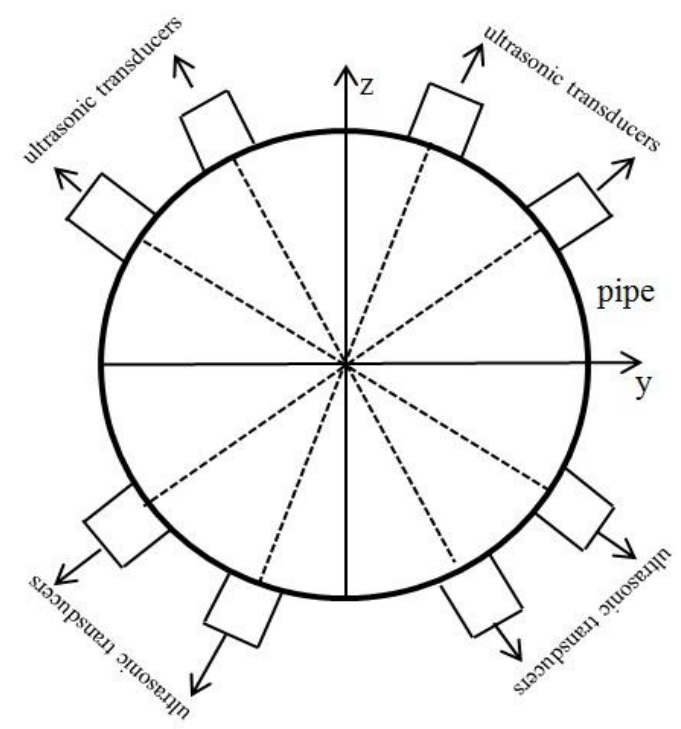

Fig.5. The transducers placed around the pipe

Usually, for ultrasonic flowmeter applications in the industry, ultrasonic transducers have a design as shown in Fig. 6.

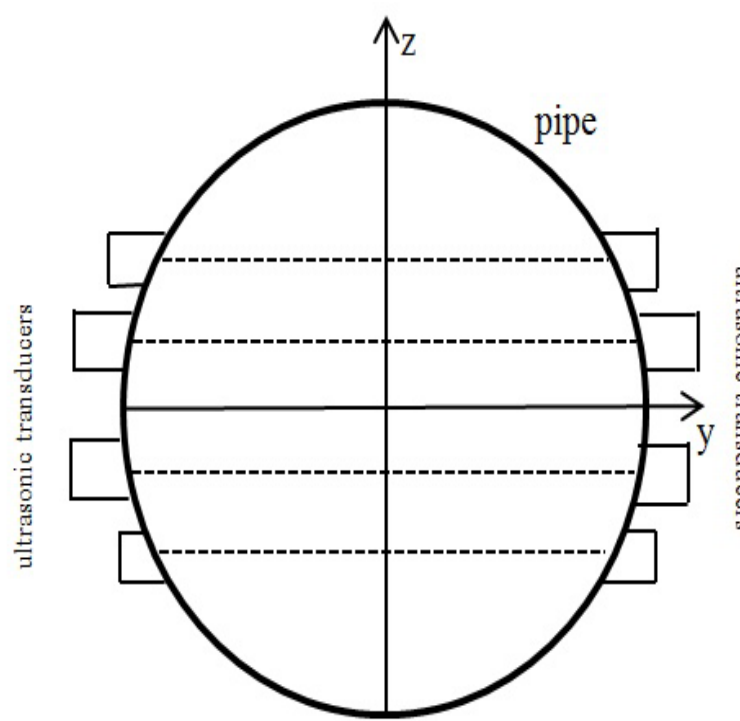

Fig.6. Ultrasonic transducers located in the z-direction

The ultrasonic transducers may be located in the middle of the pipe (as shown in Fig. 3) or at any point in the z-direction (as shown in Fig. 6). Thus, each transducer pair has a different velocity and acoustic path length information. In this paper, the normalized average flow velocity value of each acoustic path shown in Fig. 6 is calculated using pixels. For this purpose, the acoustic paths between the ultrasonic transducers are first illustrated by pixels as shown in Fig. 7. Instead of handling the entire distance between two transducers, only the pixels between the ultrasonic transducer $A$ and center (as shown in Fig. 3) are handled to avoid extra processing.

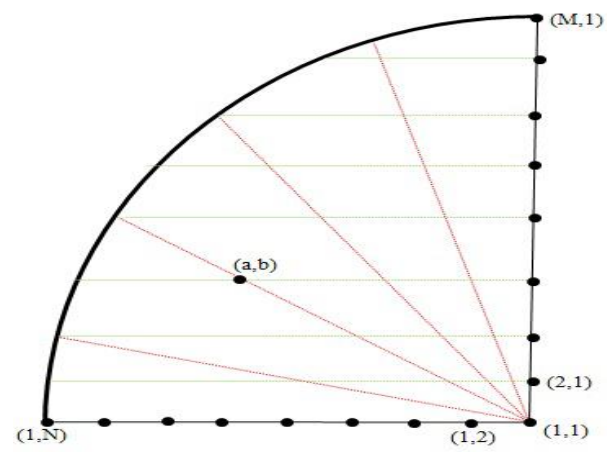

Fig.7. Finding pixel value on the acoustic path at any point in the $z$ direction

The pixel values of the red acoustic paths can be calculated as previously stated in this paper. The green acoustic paths indicate the acoustic paths between the ultrasonic transducers located in the z-direction as shown in Fig. 6. While $\mathrm{N}$ represents the number of pixels in each acoustic path, $\mathrm{M}$ indicates the acoustic path number. Increasing the number of $\mathrm{M}$ and $\mathrm{N}$ means more precise result but more processing. So, in this study, $\mathrm{M}$ and $\mathrm{N}$ values are chosen as 1000 (that means the image shown in Fig. 7 has 1000*1000 pixels). a and b are the vertical and horizontal positions of the pixel to be calculated, respectively. To find the pixel $(\mathrm{a}, \mathrm{b})$ value, the following algorithm is applied:

- Calculating the transducer distance $=\sqrt{r_{o}^{2}-(a-1)^{2}}$

- Calculating the point distance $=$

$$
\text { transducer distance } * \frac{b-1}{r_{0}}
$$

- Calculating the $\mathrm{r}$ distance $=$

$$
\sqrt{(\text { point distance })^{2}+(a-1)^{2}}
$$

$\cdot v(\mathrm{a}, \mathrm{b})=v_{m}\left(1-\frac{\mathrm{r} \text { distance }}{\mathrm{r}_{0}}\right)^{p}$

where $\mathrm{v}(\mathrm{a}, \mathrm{b})$ is the pixel value at $(\mathrm{a}, \mathrm{b})$. Transducer distance, point distance, and $r$ distance shown in Eqs. (8-11) are indicated in Fig. 8:

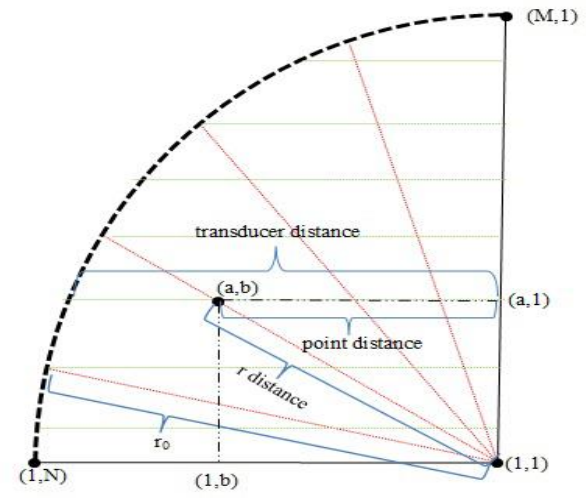

Fig.8. Transducer distance, point distance, and $\mathrm{r}$ distance

The lengths of the vertical position and horizontal position of the pixel $(a, b)$ are (a-1) and (b-1), respectively. The value 
of the $r_{0}$ is $M-1$ or $\mathrm{N}-1$. All pixel values on the green acoustic paths shown in Fig. 7 can be calculated by using Eqs. (8-11) to obtain FMIFC. The $\mathrm{r}$ distance is calculated using Eqs. (8-10) to obtain any $\mathrm{v}(\mathrm{a}, \mathrm{b})$ value in the image. For example, let the image has $10 * 10$ pixels and calculate the $\mathrm{r}$ distance for $\mathrm{v}(3,8)$. In this case, the transducer distance is the distance from $(3,10)$ to $(3,1)$, while the point distance is the distance from $(3,8)$ to $(3,1)$. Thus the $r$ distance is the distance from $(3,8)$ to $(1,1)$. After calculating the $r$ distance, $v(a, b)$ value is obtained using Eq. (11). $v_{m}$ which is pixel value at $(1,1)$ is 255 . As stated in Eq. (6), parameter p shown in Eq. (11) depends on the Re. Thus, FMIFC can be obtained for each Re. Fig. 9 shows FMIFC having $1000 * 1000$ pixels for $\mathrm{Re}=100000$.

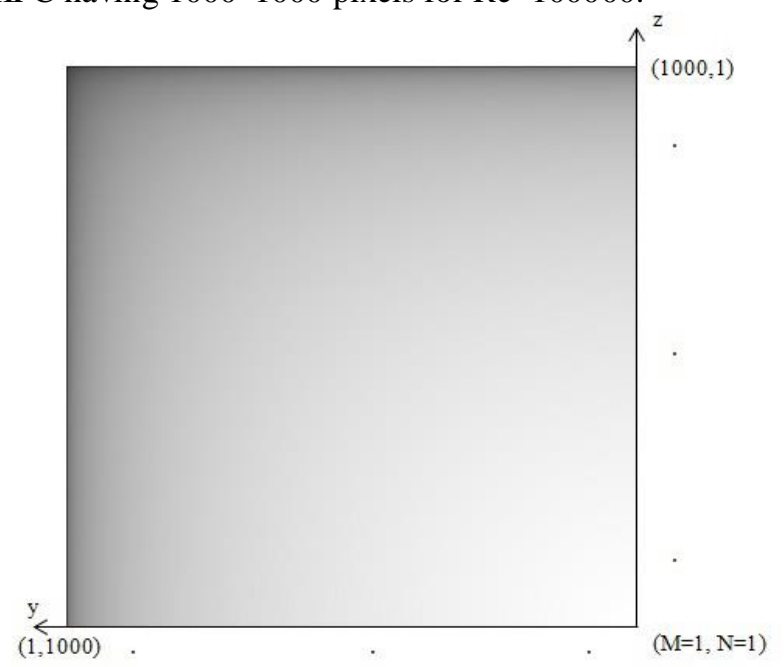

Fig.9. FMIFC for $\mathrm{Re}=100000$

Fig. 9 is the diagram showing the rectangular form of Fig. 7 after calculating the pixels on the acoustic paths shown in Fig. 7. There are 1000 acoustic paths and each acoustic path has 1000 pixels in Fig. 9. The pixel at $(\mathrm{M}=1, \mathrm{~N}=1)$ corresponds to the $\mathrm{v}_{\mathrm{m}}$. The pixels at $\mathrm{M}=1000$ or $\mathrm{N}=1000$ correspond to the pipe surface. FMIFC can be used to analyze fluid flow for different Re values. Besides, FMIFC can be used to obtain the ideal average fluid velocity ratio between the acoustic paths in the $\mathrm{z}$-direction. The average fluid velocity, for any acoustic path in the $\mathrm{z}$-direction, is calculated by averaging the obtained pixels on this acoustic path. Thus, a quality metric is obtained in this paper, which indicates the ratio of the average fluid velocities of different acoustic paths. The quality metric based on pixel $\left(\mathrm{Q}_{\mathrm{bp}}\right)$ is calculated as follows:

$$
Q_{b p}=\frac{Q_{b p r}}{Q_{a}}
$$

where $\mathrm{Q}_{\mathrm{bpr}}$ and $\mathrm{Q}_{\mathrm{a}}$ are the quality metrics for reference and application, respectively and defined as

$$
Q_{b p r}=\frac{v_{b p 1}}{v_{b p 2}}
$$

$$
Q_{a}=\frac{v_{1}}{v_{2}}
$$

where $\mathrm{v}_{\mathrm{bp} 1}$ and $\mathrm{v}_{\mathrm{bp} 2}$ are the normalized average fluid velocities based on pixel for reference, $v_{1}$ and $v_{2}$ are the average fluid velocities for the application. A researcher places the ultrasonic transducers in any positions along the $\mathrm{z}$-direction on the pipe. To obtain two different flow velocities, $v_{1}$ and $v_{2}$, from two different transducer pairs, the average fluid flow velocity on the acoustic path between each ultrasonic transducer pair is measured for turbulent flow and ideal flow conditions. $v_{b p 1}$ and $v_{b p 2}$ are obtained by the method mentioned above for reference. If there is problem such as the placement of transducers in the design, the ratio between $\mathrm{Q}_{b p r}$ and $\mathrm{Q}_{\mathrm{a}}$ is different from 1. That the ideal value of $\mathrm{Q}_{b p}$ is 1 indicates excellent compatibility between the transducer pairs. Thus, this metric can be used to assess the quality of the ultrasonic flowmeter for turbulent flow.

\section{RESULTS AND DISCUSSION}

In this paper, FMIFC is obtained in MATLAB environment to analyze fluid flow for different Re values. Fig. 10 shows FMIFC for $\mathrm{Re}=10000,100000$ and 1000000 .

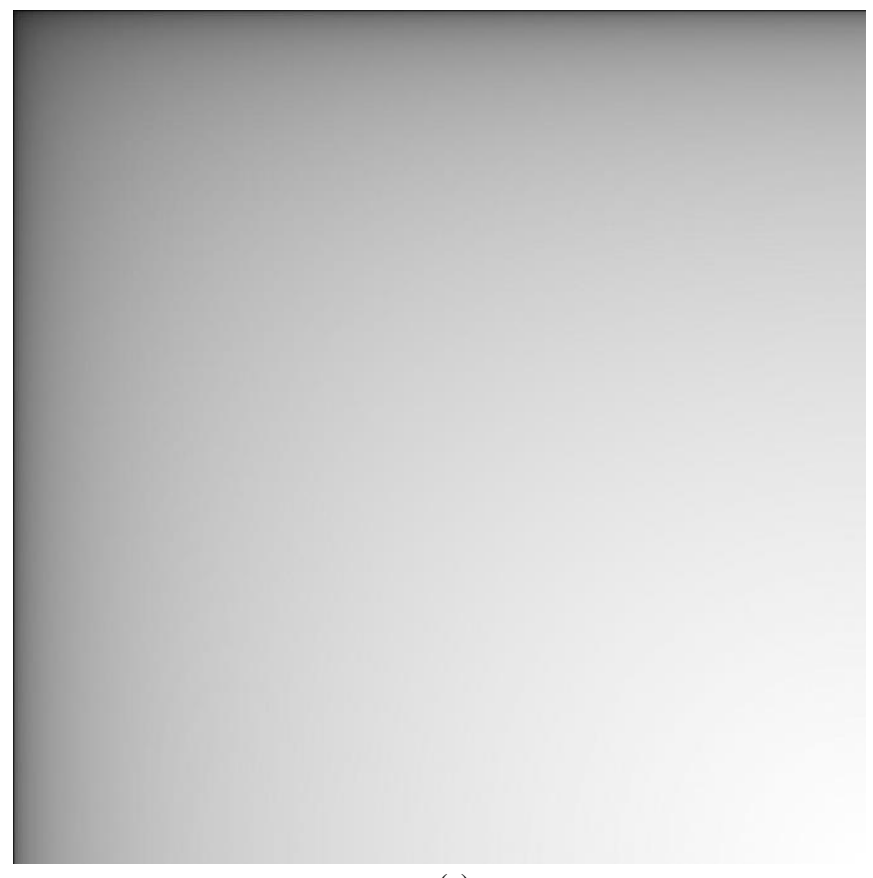

(a) 


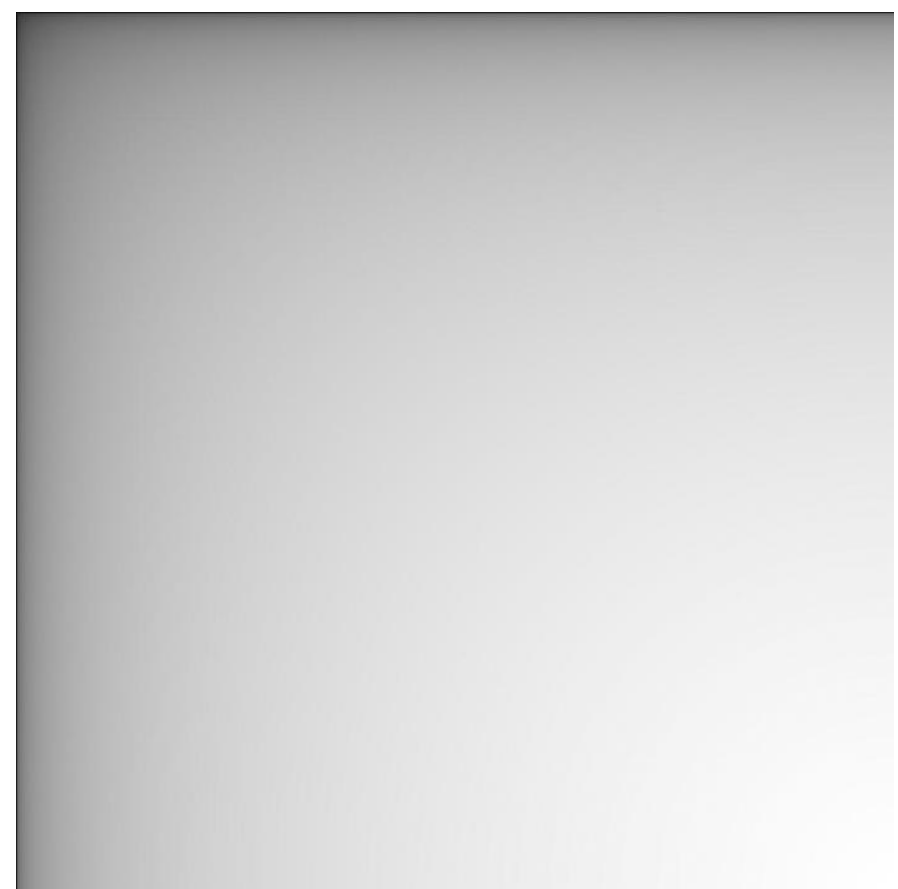

(b)

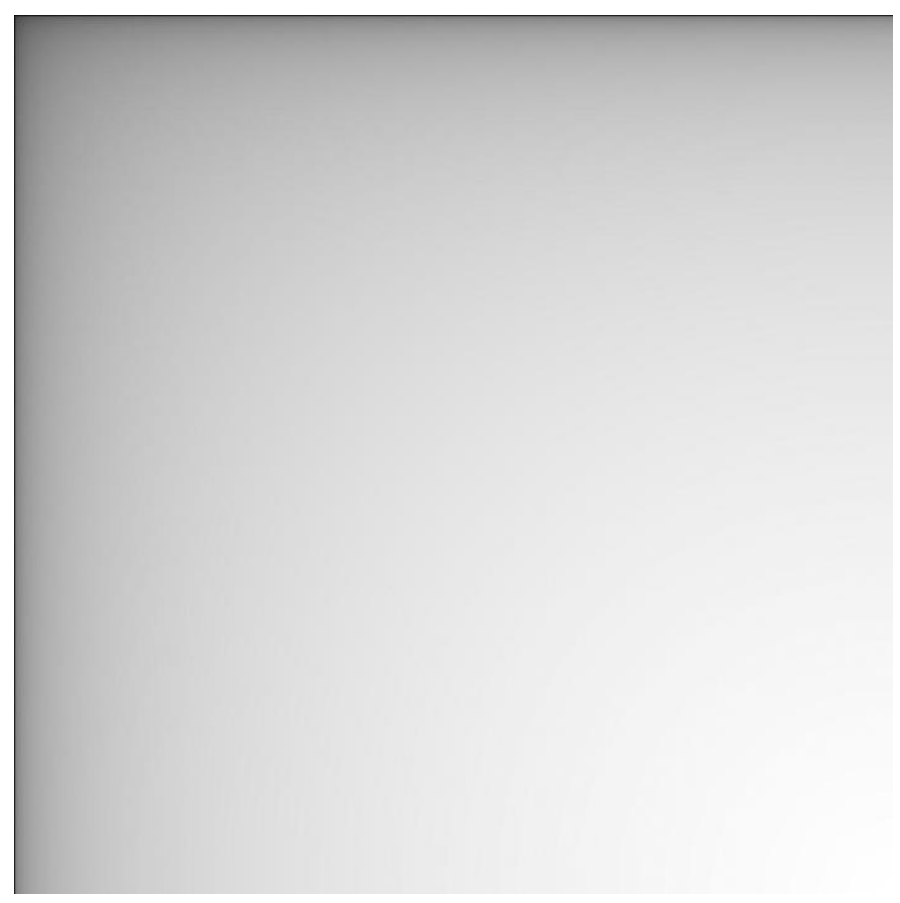

(c)

Fig.10. FMIFC for Re is (a) 10000 (b) 100000 (c) 1000000

As mentioned above, subfigures in Fig. 10 have $1000 * 1000$ pixels (i.e., they have 1000 acoustic paths and each acoustic path has 1000 pixels). The value of the pixel at $(\mathrm{M}=1, \mathrm{~N}=1)$ is 255 for each figure. Comparing the subfigures in Fig. 10, FMIFC has whiter pixels for the larger Re. That means any acoustic path in the z-direction has greater velocity (larger pixel value) for larger Re. By using FMIFC, the normalized fluid velocities are obtained for $\mathrm{M}=1,500,1000$ and $\mathrm{Re}=10000,100000$ and 1000000 (shown in Fig. 11).

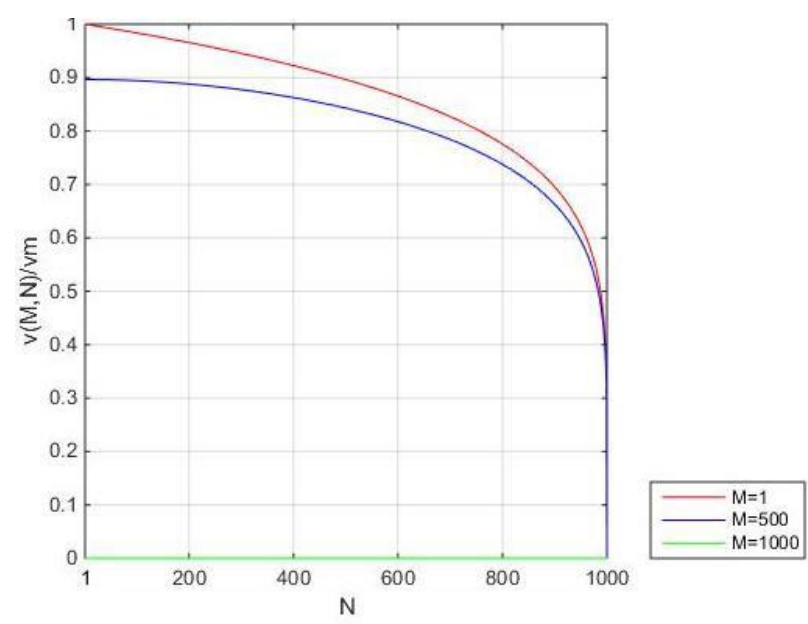

(a)

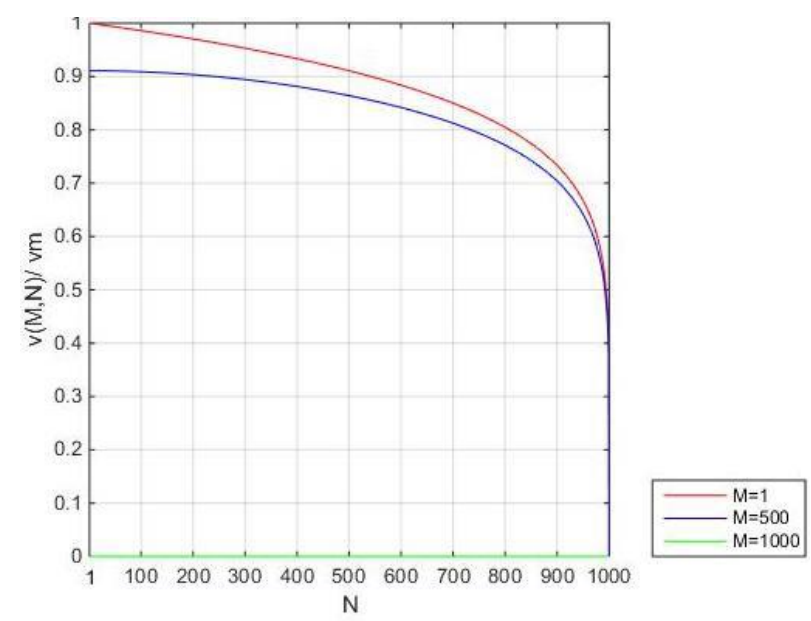

(b)

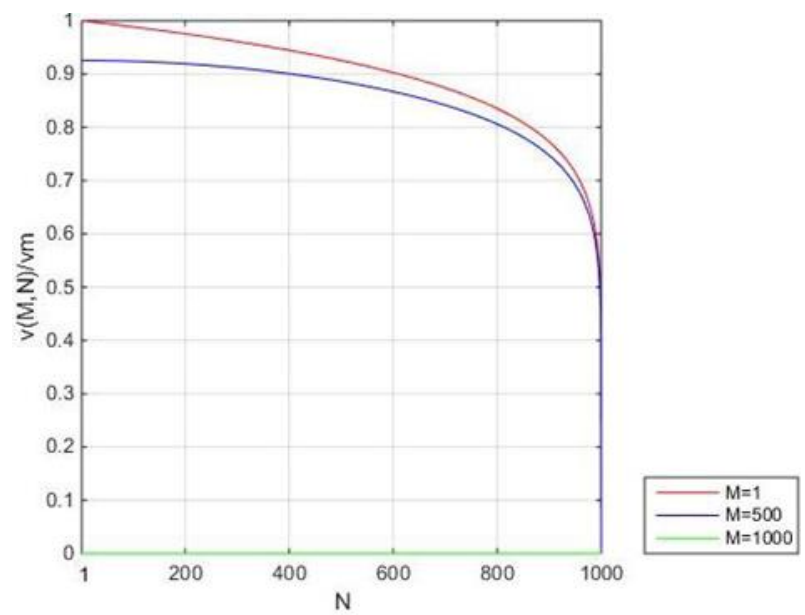

(c)

Fig.11. Normalized fluid velocity for $\mathrm{M}=1,500$ and 1000. (a) $\mathrm{Re}=10000$ (b) $\mathrm{Re}=100000$ (c) $\mathrm{Re}=1000000$

$\mathrm{v}(\mathrm{M}, \mathrm{N})$ shown in Fig. 11 indicates the pixel value of the fluid velocity at the position $(\mathrm{M}, \mathrm{N})$. The $\mathrm{v}(1,1)$ value (i.e. $\mathrm{M}=1$ and $\mathrm{N}=1)$ is equal to $\mathrm{v}_{\mathrm{m}}$ in three figures. While normalized $\mathrm{v}(500,1)$ is 0.896 for $\mathrm{Re}=10000$, it is 0.911 and 
0.925 for $\mathrm{Re}=100000$ and 1000000 , respectively. The pixels at $\mathrm{M}=1$ or $\mathrm{N}=1$ are also calculated with Eq. (5) but other pixels are calculated with pixel-based calculation method. To find normalized $\mathrm{v}(1,600)$ (whose values are $0.865,0.884$ and 0.903 for $\mathrm{Re}=10000,100000$ and 1000000 , respectively), both Eq. (5) and pixel-based method are used. To find normalized v $(500,600)$ (whose values are $0.818,0.842$ and 0.867 for $\mathrm{Re}=10000,100000$ and 1000000, respectively), only the pixelbased method is used in this paper. $\mathrm{M}=1000$ or $\mathrm{N}=1000$ indicates the pipe surface and the values of $\mathrm{v}$ are zero for these positions. Fig. 11 is also used to calculate $\mathrm{Q}_{\mathrm{bpr}}$ which is indicated in Eq. (13). For any acoustic path in the z-direction, $\mathrm{v}_{\mathrm{bp}}$ is obtained by averaging the pixels. Fig. 12 shows the obtained $\mathrm{v}_{\mathrm{bp}}$ values for different acoustic paths.

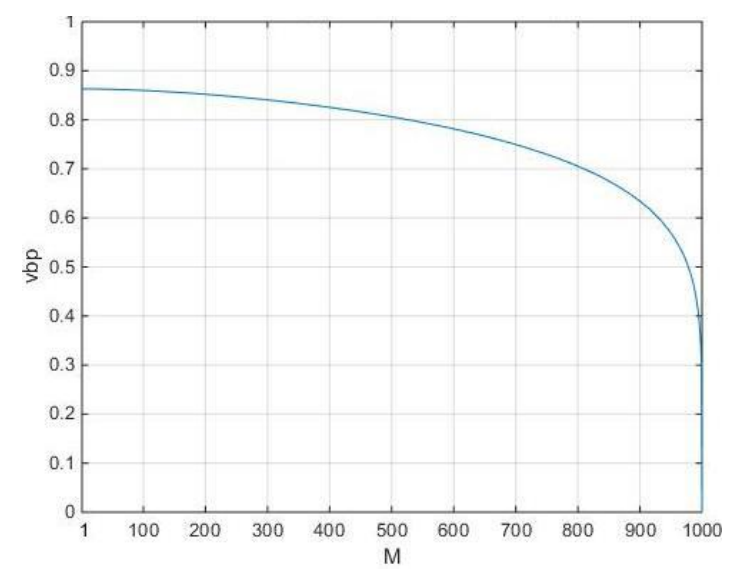

(a)

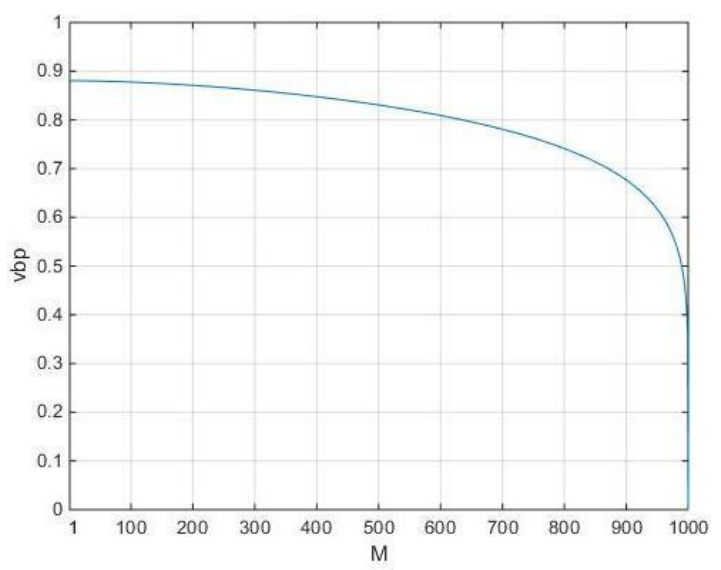

(b)

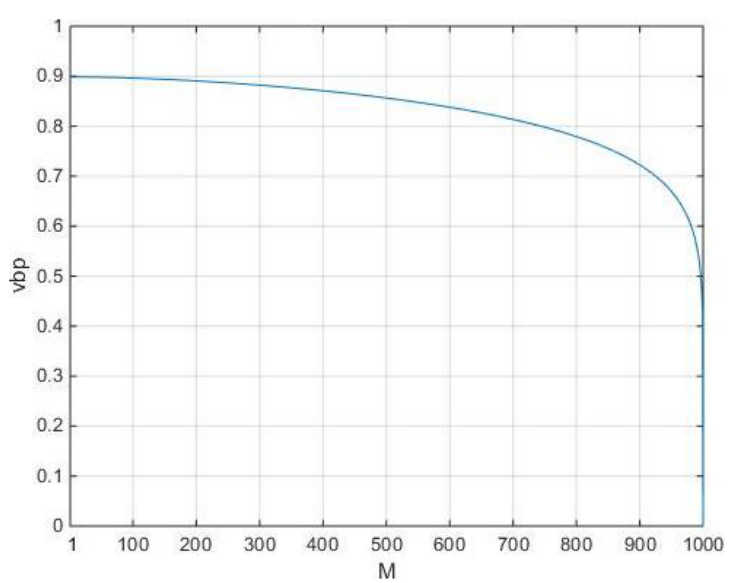

(c)

Fig.12. Obtained $v_{b p}$ values for different acoustic paths (a) $R e=10000$ (b) $\operatorname{Re}=100000$ (c) $\mathrm{Re}=1000000$

In Fig. 12, M=1 indicates the acoustic path in the middle of the pipe. In this position, $\mathrm{v}_{\mathrm{bp}}$ is $0.863,0.881$ and 0.899 for $\operatorname{Re}=10000,100000$ and 1000000 , respectively. These values are also shown in Fig. 4 and can also be obtained with Eq. (7). But for other acoustic paths, the values of $v_{b p}$ are obtained with the pixel-based calculation method. These values are used to evaluate the quality of the ultrasonic flow meter systems. If the ultrasonic transducers are placed in positions $\mathrm{M}=309$ and $\mathrm{M}=809$, we obtain that $\mathrm{v}_{\mathrm{bp} 1}$ is 0.839 for the position $\mathrm{M}=309$ and $\mathrm{v}_{\mathrm{bp} 2}$ is 0.7 for the position $\mathrm{M}=809$ as shown in Fig. 12a for $\mathrm{Re}=10000$. According to Eq. (13), $\mathrm{Q}_{\mathrm{bpr}}$ is 1.199. To evaluate the design, $\mathrm{Q}_{\mathrm{bpr}}$ is used for reference value. The researcher calculates $Q_{b p}$ by using the results of the application ( $v_{1}$ and $v_{2}$ shown in Eq. (14)). The value of $\mathrm{Q}_{\mathrm{bp}}$ shows the compatibility between the ultrasonic transducers. The ideal value of $\mathrm{Q}_{\mathrm{bp}}$ is 1 . For example, the researcher designs the ultrasonic flowmeter and calculates $Q_{b p}$ with the method given in this paper, and then changes the design and recalculates $\mathrm{Q}_{\mathrm{bp}}$. If the value of the recalculated $\mathrm{Q}_{b p}$ is closer to 1 after the operation, this means the change has improved the design. Otherwise, the change has damaged the performance of the design. Thus, $\mathrm{Q}_{\mathrm{bp}}$ can be used to analyze the design for any Re and acoustic path.

\section{CONCLUSION}

TTD method measures the average velocity of the fluid along the acoustic path between the two ultrasonic transducers. Currently, there are many studies on TTD ultrasonic flowmeters. For researchers, it is very important to analyze their designs. This paper presents FMIFC to analyze the fluid flow depending on $\mathrm{Re}$ for the ideal flow conditions and the turbulent flow. FMIFC is obtained with the pixels describing the acoustic paths. FMIFC in this paper has $1000 * 1000$ pixels which represent the number of acoustic paths and the number of pixels in each acoustic path. Any pixel in FMIFC has a greater grey level value for greater velocity. Furthermore, a quality metric is developed by using the obtained FMIFC. This metric indicates the reference ratio of the average fluid velocity of different acoustic paths. The results show that the proposed metric is easy to use for any acoustic path or Re. 
Thus, it is recommended as a useful alternative metric to evaluate the performance of the ultrasonic flowmeter.

\section{REFERENCES}

[1] Y. Inoue, H. Kikura, H. Murakawa, M. Aritomi, M. Mori, "A study of ultrasonic propagation for ultrasonic flow rate measurement.", Flow Measurement and Instrumentation, vol.19. 3-4, 2008, pp. 223-232.

[2] Y. Yu, G. Zong, "Design and simulation of an ultrasonic flow meter for thin pipe", In 2011 IEEE International Conference on Mechatronics and Automation, August 2011, pp. 1115-1119.

[3] L. Mingwei, L. Guosheng, H. Yanguo, "Research on improving the accuracy of the ultrasonic flow-meter with time difference method", In 2010 International Conference on Electrical and Control Engineering, June 2010, pp. 1704-1707.

[4] L. Svilainis, P. Kabisius, A. Aleksandrovas, A. Chaziachmetovas, "Excitation signal's influence on ultrasonic transit time flow meter's performance", In IOP Conference Series: Materials Science and Engineering, vol. 42. 1, 2012, pp. 012047.

[5] S. V. Kulkarni, M. M. Sonkhaskar, S. D. Pardeshi, "Test bench development for acquisition module FPGA of Ultrasonic flow meter", In 2015 International Conference on Pervasive Computing (ICPC), January 2015, pp. 1-6.

[6] Y. Wang, "New-type ultrasonic flow meter design based on FPGA highspeed data sampling", In 2009 9th International Conference on Electronic Measurement \& Instruments, August 2009, pp. 1-509-512.

[7] Y. Bo, C. Li, "Electronic circuit design for reciprocal operation of transit-time ultrasonic flow meters.", Flow Measurement and Instrumentation, vol.32, 2013, pp.5-13.

[8] J. Berrebi, P. E. Martinsson, M. Willatzen, J. Delsing, "Ultrasonic flow metering errors due to pulsating flow.", Flow Measurement and Instrumentation, vol. 15.3, 2004, pp. 179-185.

[9] Z. Chen, Z. Li, "Robust precise time difference estimation based on digital zero-crossing detection algorithm.", IEEE Transactions on Instrumentation and Measurement, vol. 65.8, 2016, pp.1739-1748.

[10] A. Hamouda, O. Manck, M. Hafiane, N. E. Bouguechal, "An enhanced technique for ultrasonic flow metering featuring very low jitter and offset.", Sensors, vol. 16.7, 2016, pp.1008.

[11] I. Gryshanova, I. Korobko, P. Pogrebniy, "Increasing of accuracy of multipath ultrasonic flow meters by intelligent correction.", Measurement Automation Monitoring, vol. 62,2016, pp.411-416.

[12] K. Zanker, "Diagnostic ability of the daniel four-path ultrasonic flow meter.", In Southeast Asia Flow Measurement Workshop, March 2003.

[13] T. Tresch, P. Gruber, T. Staubli, "Comparison of integration methods for multipath acoustic discharge measurements.", In proc. IGHEM, July 2006, pp. 1-16.

[14] T. Tresch, B. Lüscher, T. Staubli, P. Gruber, "Presentation of optimized integration methods and weighting corrections for the acoustic discharge measurement.", In International conference on hydraulic efficiency measurements, September 2008.

[15] A. Voser, "Analyse und Fehleroptimierung der mehrpfadigen akustischen Durchflussmessung in Wasserkraftanlagen", Doctoral dissertation, ETH Zurich, 1999.

[16] L. Qin, L. Hu, K. Mao, W. Chen, X. Fu, "Flowrate Determination for Arbitrary Multipath Arrangement Based on Generalized Inverse of Matrix.”, IEEE Sensors Journal, vol.17.12, 2017, pp. 3625-3634.

[17] X. Tang, X. Xie, H. Zhang, H. Zhou, "Data integration for multi-path ultrasonic flowmeter based on Levenberg-Marquardt algorithm.", IET Science, Measurement \& Technology, vol.9.8, 2015, pp. 909-920.

[18] L. Peng, B. Zhang, H. Zhao, S. A. Stephane, H. Ishikawa, K. Shimizu, "Data integration method for multipath ultrasonic flowmeter.", IEEE Sensors Journal, vol.12.9, 2012, pp. 2866-2874.

[19] L. C. Lynnworth, Ultrasonic Measurements for Process Control, Academic Press, Inc., 1989.

[20] J. T. Davies, Turbulence Phenomena, Academic Press, New York, 1972.

[21] B. Iooss, C. Lhuillier, H. Jeanneau, "Numerical simulation of transit-time ultrasonic flowmeters: uncertainties due to flow profile and fluid turbulence.", Ultrasonics, vol.40.9, 2002, pp. 1009-1015.

[22] G. Jossinet, "Mesure du debit des liquides par debitmetre accrochable a ultrasons.", NT EDF/DER, HP-12/91.26, 1991.

\section{BIOGRAPHY}

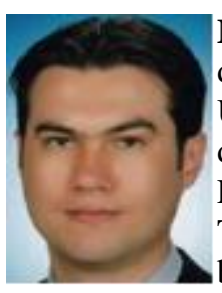

Murat Alparslan Gungor received the B.S. degree in Electronics Engineering from Istanbul University, Istanbul, in 2000, M.S. and Ph. D degrees in Electrical and Electronics Engineering from Gazi University, Ankara, Turkey, in 2008 and 2015, respectively. He became the Assistant Professor at Hitit

University in 2015. He is currently Assistant Professor in the Electrical and Electronics Engineering Department of Hitit University. His research interests include signal processing, image processing, medical imaging systems and ultrasonic flowmeters. 